\title{
A!
}

This is an electronic reprint of the original article.

This reprint may differ from the original in pagination and typographic detail.

Ermolova, Natalia; Tirkkonen, Olav

\section{SIR Statistics and Average Rates in Cooperative Spectrum Sharing Networks}

Published in:

IEEE Transactions on Vehicular Technology

DOI:

10.1109/TVT.2019.2921251

Published: 06/06/2019

Document Version

Peer reviewed version

Please cite the original version:

Ermolova, N., \& Tirkkonen, O. (2019). SIR Statistics and Average Rates in Cooperative Spectrum Sharing Networks. IEEE Transactions on Vehicular Technology, 68(8), 8178-8182.

https://doi.org/10.1109/TVT.2019.2921251

This material is protected by copyright and other intellectual property rights, and duplication or sale of all or part of any of the repository collections is not permitted, except that material may be duplicated by you for your research use or educational purposes in electronic or print form. You must obtain permission for any other use. Electronic or print copies may not be offered, whether for sale or otherwise to anyone who is not an authorised user. 


\title{
SIR Statistics and Average Rates in Cooperative Spectrum-Sharing Networks
}

\author{
Natalia Y. Ermolova, and Olav Tirkkonen, Member, IEEE \\ Department of Communications and Networking, Aalto University, P.O. Box 15500, FI-00076 Aalto, Finland, \\ e-mail: natalia.ermolova@aalto.fi; olav.tirkkonen@aalto.fi
}

\begin{abstract}
In this paper, we analyze cooperative wireless networks (primary networks) operating under randomly located interfering nodes (secondary networks). Applying Poisson point processes to modeling of network and interfering node locations, we derive statistics of signal-to-interference ratio (SIR) under different operational scenarios and show that the SIR statistical models can be viewed as special cases of the $H$-function distribution. On this basis, we obtain formulas for the average information rates. The presented results are general, and they are applicable to arbitrary fading conditions.
\end{abstract}

Index Terms-Average rate, cooperative communications, fading, interference, Fox $\mathbf{H}$ function, $\mathbf{H}$-function distribution, Poisson point process, spectrum sharing, wireless networks.

\section{INTRODUCTION}

Spectrum sharing is an important technological trend for $5 \mathrm{G}$, which aims at efficient alleviation of spectrum scarcity problem. The method assumes a coexistence of a few independent networks operating simultaneously within the same frequency band, and it can be implemented via various operational protocols under both theoretical and practical maintenance [1], [2]. The crucial problem in spectrum sharing systems is provision of an acceptable interference level for the primary network in the sense of deteriorating its performance metrics only until prerequisite levels. The signal-to-interference (SIR) statistical distribution is the key factor affecting various performance metrics of the primary network that can be used as criteria in solving this problem. Thus, SIR statistics are of large interest in design considerations of spectrum sharing networks. Among different performance metrics, the attainable average rate is one of the most important characteristics.

Stochastic geometry provides convenient and effective mathematical methods for analyzing different types of wireless networks, and SIR statistics under various operational scenarios were analyzed e.g. in [3]- [9]. These works considered the mainly cellular-like networks where the probe receiver (Rx) communicated with the strongest or closest transmitter (Tx), and the other Txs created interference. This scenario differs from that of cooperative primary network and independent interfering (secondary) network analyzed in our work. Additionally, previous analyses were restricted by Rayleigh fading only. Meanwhile, the Rayleigh fading model very often does not fit to practical measurements, and more sophisticated fading distributions showing much better agreements with real data must be used in analysis for obtaining reliable estimates [10], [11].

To the best of our knowledge, neither previous work analyzed attainable data rates in random spectrum sharing networks with arbitrary fading models of transmission links. The absence of results in this area motivated this work.

The main contributions of this paper can be summarized as follows.

- We obtain SIR statistics (in terms of probability density, cumulative distribution, and moment generating functions (PDF, CDF, and MGF)) in random cooperative spectrum sharing networks under two cooperation protocols where the cooperative Txs use either one information channel for communication with the probe RX, or they employ orthogonal channels, e.g. time-division multiplexing [12]. We prove that for arbitrary fading models of transmission links, the SIR statistical models can be viewed as special cases of the $\mathrm{H}$ function distribution [13].

-Based on the derived SIR statistical models, we analyze the achievable average information rates for different operational scenarios.

-For interference-limited scenarios, we derive closed-form expressions for the attainable information rates.

-For interference-plus-noise scenarios, we present integralform expressions and simple approximations.

The presented results can be used for both design and analysis of random spectrum sharing networks.

\section{Preliminaries}

\section{A. Fox H-function and the H-function distribution}

The $H$-function is most frequently specified via its Mellin transform, which can be represented as the ratio of products of gamma functions. Thus, the $\mathrm{H}$-function can be defined via a Mellin-Barnes integral as

$$
\begin{gathered}
H_{p, q}^{m, n}\left[x \mid \begin{array}{c}
\left(a_{1}, A_{1}\right), \ldots,\left(a_{p}, A_{p}\right) \\
\left(b_{1}, B_{1}\right), \ldots,\left(b_{q}, B_{q}\right)
\end{array}\right]=\frac{1}{2 \pi i} \int_{\mathcal{C}} x^{-s} \\
\times \frac{\prod_{j=1}^{m} \Gamma\left(b_{j}+B_{j} s\right) \prod_{j=1}^{n} \Gamma\left(1-a_{j}-A_{j} s\right)}{\prod_{j=m+1}^{q} \Gamma\left(1-b_{j}-B_{j} s\right) \prod_{j=n+1}^{p} \Gamma\left(a_{j}+A_{j} s\right)} d s
\end{gathered}
$$

where $0 \leq m \leq q$ and $0 \leq n \leq p$ are integers, $A_{j}>0$, $j=1, \ldots, p, B_{j}>0, j=1, \ldots, q, \Gamma($.$) is the gamma$ function, and $\mathcal{C}$ is a contour in the complex plane, see [13], [14] for details. The Fox $H$ - function is not available via the standard software, but routines for its evaluation are well known [15], [16]. Additionally, the Fox $H$-function reduces to the Meijer $G$-function for rational values of $A_{j}$ and $B_{j}$ [17, vol. 3, eq. (8.4.1.22)]. Since the Meijer $G$-function is implemented in modern software packages, this representation makes $H$-function applications rather general and convenient for many research and practical purposes. 
A random variable (RV) $X_{\mathrm{H}} \geq 0$ follows the $H$-function distribution if its PDF $f_{X_{\mathrm{H}}}(x)$ can be expressed via the Fox $H$-function as

$$
f_{X_{\mathrm{H}}}(x)=\kappa H_{p, q}^{m, n}\left[\mu x \mid \begin{array}{c}
\left(a_{1}, A_{1}\right), \ldots,\left(a_{p}, A_{p}\right) \\
\left(b_{1}, B_{1}\right), \ldots,\left(b_{q}, B_{q}\right)
\end{array}\right]
$$

where $\kappa, \mu, \mathbf{a}=\left\{a_{1}, \ldots, a_{p}\right\}, \mathbf{A}=\left\{A_{1}, \ldots, A_{p}\right\}, \mathbf{b}=$ $\left\{b_{1}, \ldots, b_{q}\right\}$, and $\mathbf{B}=\left\{B_{1}, \ldots, B_{q}\right\}$ are parameters.

The CDF and MGF corresponding to (2) can also be expressed in terms of $H$-function [13], [14]. We show below that SIR statistical models in cooperative spectrum sharing networks can also be viewed as special cases of (2).

\section{B. The Symmetrical $\alpha$-stable Distribution}

The symmetrical $\alpha$-stable distribution aka the one-sided Levy stable distribution is most frequently specified via the MGF. An RV $X_{\text {st }}$ follows the symmetrical $\alpha$-stable distribution if the MGF $\mathcal{M}_{X_{\text {st }}}(s) \triangleq \mathcal{L}\left\{f_{X_{\text {st }}}(x) ;\{x, s\}\right\}$ (where $\mathcal{L}$ means the Laplace transform, and $f_{X_{\mathrm{st}}}(x)$ is the PDF), can be represented as

$$
\mathcal{M}_{X_{\text {st }}}(s)=\exp \left(-K s^{\alpha}\right)
$$

where $K$ and $\alpha$ are parameters of the distribution.

A PDF expression of the $\alpha$-stable RV is less recognized. The symmetrical $\alpha$-stable distribution can, however, be viewed as a special case of the $H$-function distribution since the PDF corresponding to (3) can be represented in terms of the $\mathrm{H}$ function as [14, eq. (2.28)], [13, eq. (2.2)]

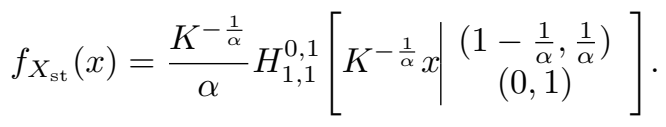

\section{SIR STATISTICS IN COOPERATIVE RANDOM NETWORKS WITH RANDOMLY LOCATED INTERFERERS}

\section{A. System Model}

We analyze a random set $\Theta_{\mathrm{T}}$ of wireless nodes (the primary network) that cooperatively communicate with a probe $\mathrm{Rx}$ under interference coming from another (secondary) spectrum sharing network $\Theta_{\mathrm{I}}$, which is also composed of randomly located nodes. We assume that the primary and secondary network nodes form homogeneous Poisson fields. Let the primary PPP be characterized by the transmit power $P_{\mathrm{T}}$ and density $\lambda_{\mathrm{T}}$, and the secondary PPP be characterized by the transmit power $P_{\mathrm{I}}$ and density $\lambda_{\mathrm{I}}$. Let the Rx of interest be located at the origin, and the networks operate in the twodimensional Euclidean space $\mathbb{R}^{2}$. We assume a conventional distance-dependent path-loss model $l(x)=L_{0}\|x\|^{-\eta}$, where \|. .| means the Euclidean distance between the Tx and Rx, $L_{0}$ represents the path loss at $\|x\|=1$, and $\eta$ is the path-loss exponent, $2<\eta \leq 6$ [10].

Then the SIR of the Tx located at $x_{j} \in \Theta_{\mathrm{T}}$ can be specified as

$$
\operatorname{SIR}_{x_{j}}=\frac{P_{\mathrm{T}} g_{x_{j}}\left\|x_{j}\right\|^{-\eta}}{\sum_{y_{j} \in \Theta_{\mathrm{I}}} P_{\mathrm{I}} g_{y_{j}}\left\|y_{j}\right\|^{-\eta}}
$$

where $g_{x_{j}}\left(g_{y_{j}}\right)$ represents the channel power gain between the Tx at $x_{j}$ (interferer at $y_{j}$ ) and Rx. We assume below that
$g_{x_{j}}=g_{\mathrm{T}}$ and $g_{y_{j}}=g_{\mathrm{I}}$. The analysis of this work holds for arbitrary statistics of $g_{\mathrm{T}}$ and $g_{\mathrm{I}}$.

We analyze operational protocols where the network nodes use either orthogonal channels for transmission (e.g., timedivision multiplexing), or all transmitting nodes use the same radio channel [12]. Under the former scenarios, the SIR CDF $F_{\mathrm{o}}(z)$ can be expressed as

$$
F_{\mathrm{o}}(z)=\operatorname{Pr}\left\{\max _{x_{j} \in \Theta_{\mathrm{T}}} \operatorname{SIR}_{x_{j}} \leq z\right\},
$$

and for the latter cases, the $\mathrm{CDF} F_{\mathrm{n} / \mathrm{o}}(z)$ can be expressed as

$$
F_{\mathrm{n} / \mathrm{o}}(z)=\operatorname{Pr}\left\{\sum_{x_{j} \in \Theta_{\mathrm{T}}} \operatorname{SIR}_{x_{j}} \leq z\right\} .
$$

\section{B. $C D F$ and PDF Expressions}

We start with analyzing SIR statistics specifying by (6).

Proposition 1: The PDF $f_{\mathrm{o}}(z)$ and $\mathrm{CDF} F_{\mathrm{o}}(z)$ can be represented as

$$
\begin{gathered}
f_{\mathrm{o}}(z)=\Xi^{-\frac{\eta}{2}} \frac{\eta}{2} H_{2,1}^{1,1}\left[\Xi^{-\frac{\eta}{2}} z \mid \begin{array}{c}
\left(-\frac{\eta}{2}, \frac{\eta}{2}\right),(-1,1) \\
\left(-\frac{\eta}{2}, \frac{\eta}{2}\right)
\end{array}\right], \\
F_{\mathrm{o}}(z)=\frac{\eta}{2} H_{2,1}^{1,1}\left[\Xi^{-\frac{\eta}{2}} z \mid \begin{array}{c}
\left(1, \frac{\eta}{2}\right),(1,1) \\
\left(1, \frac{\eta}{2}\right)
\end{array}\right]
\end{gathered}
$$

where $\Xi=\frac{\frac{\lambda_{\mathrm{T}}}{\lambda_{\mathrm{I}}} P_{\mathrm{T}}^{\frac{2}{\eta}} E\left\{\left(g_{\mathrm{T}}\right)^{\frac{2}{\eta}}\right\}}{\Gamma\left(1-\frac{2}{\eta}\right) P_{\mathrm{I}}^{\frac{2}{\eta}} E\left\{\left(g_{\mathrm{I}}\right)^{\frac{2}{\eta}}\right\}}$, and $E$ is the expectation.

Proof: A CDF expression $F_{\mathrm{o}}(z)=\mathbb{E}_{\frac{2}{\eta}}\left(-\Xi z^{-\frac{2}{\eta}}\right)$, where $\mathbb{E}_{\frac{2}{\eta}}($.$) is the ordinary Mittag-Leffler function [18], was de-$ rived in $[9$, eq. (8)]. By differentiation, we obtain that $f_{\mathrm{o}}(z)=\Xi z^{-\frac{2}{\eta}-1} \mathbb{E}_{\frac{2}{\eta}, \frac{2}{\eta}}\left(-\Xi z^{-\frac{2}{\eta}}\right)$, where $\mathbb{E}_{\frac{2}{\eta}, \frac{2}{\eta}}($.$) is the$ general Mittag-Leffler function [18]. Expressing $\mathbb{E}_{\frac{2}{n}}($.$) and$ $\mathbb{E}_{\frac{2}{\eta}, \frac{2}{\eta}}($.$) via the Fox H$-functions [18, eqs. (8.12), (8.13)] and using transformations [13, eqs. (2.2), (2.3)], we obtain (8), (9).

The following proposition is valid for SIR statistics if the cooperative primary Txs use one transmission channel.

Proposition 2: The PDF $f_{\mathrm{n} / \mathrm{o}}(z)$ and $\mathrm{CDF} F_{\mathrm{n} / \mathrm{o}}(z)$ can be represented as

$$
\begin{gathered}
f_{\mathrm{n} / \mathrm{o}}(z)=\frac{\eta^{2}}{4} \Upsilon H_{2,2}^{1,1}[\Upsilon z \\
F_{\mathrm{n} / \mathrm{o}}(z)=\frac{\eta^{2}}{4} H_{3,3}^{1,2}\left[\begin{array}{c}
\left(1-\frac{\eta}{2}, \frac{\eta}{2}\right),(-1,1) \\
\left(-\frac{\eta}{2}, \frac{\eta}{2}\right),(0,1)
\end{array}\right], \\
\left.\Upsilon z \mid \begin{array}{l}
(1,1),\left(1, \frac{\eta}{2}\right),(0,1) \\
\left(0, \frac{\eta}{2}\right),(1,1),(0,1)
\end{array}\right]
\end{gathered}
$$

where $\Upsilon=\frac{P_{\mathrm{I}}}{P_{\mathrm{T}}}\left(\frac{\lambda_{\mathrm{I}} E\left\{\left(g_{\mathrm{I}}\right)^{\frac{2}{\eta}}\right\}}{\lambda_{\mathrm{T}} E\left\{\left(g_{\mathrm{T}}\right)^{\frac{2}{\eta}}\right\}}\right)^{\frac{\eta}{2}}$.

Proof: We take into account that the SIR in (7) is the ratio of two symmetrical $\alpha$-stable RVs where the PDFs of both RVs are given by (4) with the respective parameters $K=K_{\text {us }}$ and $K=K_{\mathrm{int}}$, where $K_{\mathrm{us}}\left(K_{\mathrm{int}}\right)=$ $\pi \Gamma\left(1-\frac{2}{\eta}\right) \lambda_{\mathrm{T}}\left(\lambda_{\mathrm{I}}\right)\left[L_{0} P_{\mathrm{T}}\left(P_{\mathrm{I}}\right)\right]^{\frac{2}{\eta}} E\left\{g_{\mathrm{T}}^{\frac{2}{\eta}}\left(g_{\mathrm{I}}^{\frac{2}{\eta}}\right)\right\}$ [9, eq. (6)]. Then the ratio PDF (10) can be obtained from [13, eq. (4.13)]. Eq. (11) results from integration of (10) via [17, vol. 3, eq. (1.16.4.1)]. 


\section{Average Rates}

The average rate $\mathcal{R}$ can be specified as $\mathcal{R}=$ $\frac{1}{\ln 2} \int_{0}^{\infty} \ln (1+x) f_{\text {SINR }}(x) d x$, where SINR denotes the signal-to-interference-plus noise power ratio, and $f_{\operatorname{SINR}}(x)$ is the PDF of SINR [19].

\section{A. Interference-limited Scenarios}

Proposition 3: If the cooperative Txs use orthogonal transmission channels, the average rate can be expressed as

$$
\mathcal{R}_{\mathrm{o}}=\frac{\eta}{2 \ln 2} H_{4,3}^{3,2}\left[\begin{array}{c|c}
\Xi^{-\frac{\eta}{2}} & (0,1),\left(0, \frac{\eta}{2}\right),(0,1),(1,1) \\
(0,1),(0,1),\left(0, \frac{\eta}{2}\right)
\end{array}\right] .
$$

If all Txs use one radio channel, then the average rate can be assessed as

$$
\mathcal{R}_{\mathrm{n} / \mathrm{o}}=\frac{\eta^{2}}{4 \ln 2} H_{4,4}^{3,2}\left[\Upsilon \mid \begin{array}{l}
(0,1),\left(1, \frac{\eta}{2}\right),(0,1),(1,1) \\
(0,1),(0,1),\left(0, \frac{\eta}{2}\right),(1,1)
\end{array}\right] .
$$

Proof: To evaluate (12), (13), we use a representation $\ln (1+x)=x_{2} F_{1}(1,1,2,-x)$ [17, eq. (7.3.2.148)], where ${ }_{2} F_{1}($.$) is the Gauss hypergeometric function. Then we apply$ an integration formula [17, vol. 3, eq. (2.25.3.5)] with the PDFs $f_{\text {SIR }}(x)$ specified by (8) and (10) results in (12) and (13), respectively.

Corollary 1: Effects of fading in (8)-(13) are canceled if the channel gains $g_{\mathrm{T}}$ and $g_{\mathrm{I}}$ follow identical fading distributions.

\section{B. Interference-Plus-Noise Scenarios}

Under these scenarios, an MGF-based approach can be applied for specification of average information rates [20, eq. (2)]. The method requires knowledge on MGF expressions of the useful signal and interference.

1) Non-orthogonal Cooperative Transmission: In this case, both the useful signal and interference are symmetric $\alpha$-stable RVs, and their MGFs can be specified by (3) with $\alpha=\frac{2}{\eta}$ and with the respective parameters $K=\tilde{K}_{\text {us }}\left(\tilde{K}_{\text {int }}\right)=$ $\pi \Gamma\left(1-\frac{2}{\eta}\right) \lambda_{\mathrm{T}}\left(\lambda_{\mathrm{I}}\right)\left[L_{0} P_{\mathrm{T}}\left(P_{\mathrm{I}}\right) / \sigma^{2}\right]^{\frac{2}{\eta}} E\left\{g_{\mathrm{T}}^{\frac{2}{\eta}}\left(g_{\mathrm{I}}^{\frac{2}{\eta}}\right)\right\}$ where $\sigma^{2}$ is the the variance of additive white Gaussian noise.

Proposition 4: The average rate $\tilde{\mathcal{R}}_{\mathrm{n} / \mathrm{o}}$ can be evaluated as

$$
\begin{aligned}
\tilde{\mathcal{R}}_{\mathrm{n} / \mathrm{o}}= & \frac{1}{\ln 2} \int_{0}^{\infty} \frac{\exp \left(-\tilde{K}_{\mathrm{int}} s^{\frac{2}{\eta}}\right)\left[1-\exp \left(-\tilde{K}_{\mathrm{us}} s^{\frac{2}{\eta}}\right)\right]}{s} \\
& \times \exp (-s) d s \approx \frac{\eta}{2 \ln 2} \ln \left(1+\frac{\tilde{K}_{\mathrm{us}}}{\tilde{K}_{\mathrm{int}}+1}\right)
\end{aligned}
$$

Proof: See Appendix A.

The approximate expression in (14) represents an easy tractable formula.

2) Orthogonal Cooperative Transmission: The following proposition is valid.

Proposition 5: The average rate can be assessed as

$$
\begin{aligned}
& \tilde{\mathcal{R}}_{\mathrm{o}}=\frac{1}{\ln 2} \int_{0}^{\infty} \frac{\exp \left(-\tilde{K}_{\mathrm{int}} s^{\frac{2}{\eta}}\right) \exp (-s)}{s} \\
& \quad \times\left(1-H_{0,2}^{2,0}\left[\Theta s \mid(0,1),\left(1, \frac{\eta}{2}\right)\right]\right) d s
\end{aligned}
$$

where $\Theta=\frac{P_{\mathrm{T}} L_{0}}{\sigma^{2}}\left(\pi \lambda_{\mathrm{T}} E\left\{g_{\mathrm{T}}^{\frac{2}{\eta}}\right\}\right)^{\frac{\eta}{2}}$.

Proof: See Appendix B.

The integrals in (14), (15) can be evaluated, e.g., via the Gauss-Laguerre quadrature. Eq. (14) can also be used for assessing upper bounds on $\tilde{\mathcal{R}}_{\mathrm{o}}$. Additionally, corresponding upper bounds can be evaluated with the help of (12)-(13) assessing the average rates under interference-limited scenarios.

\section{NumERICAL EXAMPLES}

To illustrate the generality of the derived results and to analyze effects of different propagation conditions, we present numerical results for various propagation parameters. We tested effects of different fading severity and path-loss exponent values $\eta=2.7, \eta=3.8$, and $\eta=5$ corresponding to different propagation scenarios [21]. In all figures, single points represent simulation results.

Results on the OP under orthogonal cooperative transmission (9) were reported in [9], and in Fig. 1, we present OP curves (11) for scenarios where the useful and interference links follow identical fading distributions, $P_{\mathrm{T}} / P_{\mathrm{I}}=0.01$, and $\lambda_{\mathrm{T}} / \lambda_{\mathrm{I}}=\{20,50,100,200\}$. The results in Fig. 1 confirmed the statement of corollary 1. In Fig. 2, OP estimates for different fading statistics of useful and interfering links are shown versus $\lambda_{\mathrm{T}} / \lambda_{\mathrm{I}}$ for $P_{\mathrm{T}} / P_{\mathrm{I}}=10, z=10 \mathrm{~dB}$. We tested four fading scenarios for two path-loss exponent values. Under scenarios (1)-(3), we tested Nakagami- $m$ and generalized gamma (GG) fading distributions for the useful and interfering links with the respective PDFs of channel power gains $f_{g_{\mathrm{Nak}}}(x)=\frac{x^{m-1} m^{m}}{\Gamma(m) \bar{g}_{\mathrm{Nak}}^{m}} \exp \left(-x \frac{m}{\bar{g}_{\mathrm{Nak}}}\right)$ and $f_{g_{\mathrm{GG}}}=$ $\frac{\nu\left(\beta / \bar{g}_{\mathrm{GG}}\right)^{m \nu}}{\Gamma(m)} x^{\nu m-1} \exp \left[-\left(\frac{\beta x}{\bar{g}_{\mathrm{GG}}}\right)^{\nu}\right]$, where $m$ and $\nu$ are shape parameters, $\beta=\Gamma(m+1 / \nu) / \Gamma(m)$, and $\bar{g}_{\mathrm{Nak}(\mathrm{GG})}$ denotes the expectation of corresponding power gain [22]. Under scenarios (1)-(3), $m=1.7$. For the case (1), the interfering links followed the GG fading with $\nu_{\mathrm{I}}=6$ while the useful links followed Nakagami- $m$ fading, i.e., $\nu_{\mathrm{T}}=1$. Under scenario (2), both the useful and interfering links followed Nakagami- $m$ fading, and for the case (3), the useful links followed GG fading with $\nu_{\mathrm{T}}=6$, and the interfering links followed Nakagami- $m$ fading. Under scenario (4), the useful and interfering links followed Nakagami- $m$ statistical models with the respective shape parameters $m_{\mathrm{T}}=3.5$ and $m_{\mathrm{I}}=0.7$. Both shape parameters $m$ and $\nu$ are inversely proportional to the amount of fading, and thus their increasing boosts effects of corresponding links.

Average information rates achievable in the considered network are shown in Fig. 3-5. In Fig. 3, numerical estimates evaluated via (12), (13) are shown for identical statistics of useful and interfering links. In Fig. 4 and in Fig. 5, average rates were evaluated for interference-plus-noise scenario versus the signal-to-noise ratio (SNR), $P_{\mathrm{T}} / \sigma^{2}$. The analytical results in Fig. 4 were evaluated via (14). We assumed identical Nakagami- $m$ useful and interfering links with $m=2.7$. In Fig. 5, average information rates for orthogonal and nonorthogonal cooperative transmissions under interference-plusnoise scenarios are shown. We assumed identical Nakagami- $m$ useful and interfering links with $m=1.3$ and $\lambda_{\mathrm{T}} / \lambda_{\mathrm{I}}=50$. 


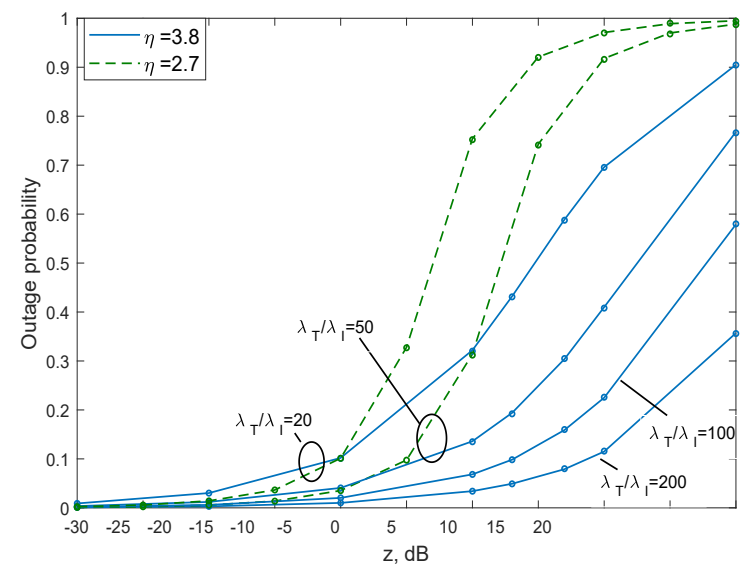

Fig. 1: Outage probability (11) for identical fading distributions of useful and interfering links. $P_{\mathrm{T}} / P_{\mathrm{I}}=0.01$.

As the parameter $m$ increases, both the useful and interfering signals boost, and the curves in Fig. 4-5 show that for the used parameter combination, the average rates slightly increase as $m$ increases. It can also be seen that the average rates for $m=$ 1.3 approach the corresponding rates for $m=2.7$ as the SNR increases (the operational conditions approach interferencelimited scenarios). This fact also confirms the statement of corollary 1 valid for interference-limited scenarios.

In our numerical results, we observed that increasing of the path-loss exponent $\eta$ had positive effects on system performance. Both the useful and interfering signal powers decrease as $\eta$ increases, and our numerical results can be explained by the parameter combination used.This observation agrees with [9, eq. (8)].

In this work, we used a method of the $H$-function implementation via its reduction to the Meijer $G$-function [17, vol. 3 , eq. (8.4.1.22)]. For all considered scenarios, we observed a good agreement between analytical and simulation results, and numerical evaluations were fast and efficient.

\section{CONCLUSION}

As spectrum sharing becomes an important tendency in $5 \mathrm{G}$ networks, theoretical and practical supports of this technology become key issues. In this paper, we obtained formulas for the SIR statistics and average rates in cooperative spectrum sharing networks assuming that both useful and interfering node locations formed PPPs.

For a few operational scenarios, we derived closedform PDF, CDF, and MGF expressions and showed that the SIR statistical models can be viewed as special cases of the $H$-function distribution. Then we applied theory of the $H$-function distributions to analysis of average information rates. For interference-limited scenarios, we obtained closed-form expressions. They can be used as upper bounds under interference-plus-noise scenarios. Additionally, for interference-plus-noise scenarios, we derived integral-form formulas and a very simple and analytically tractable approximate expression.

Many results of this work were derived in terms of Fox $H$-function. For rational values of the parameters $A_{j}$ and

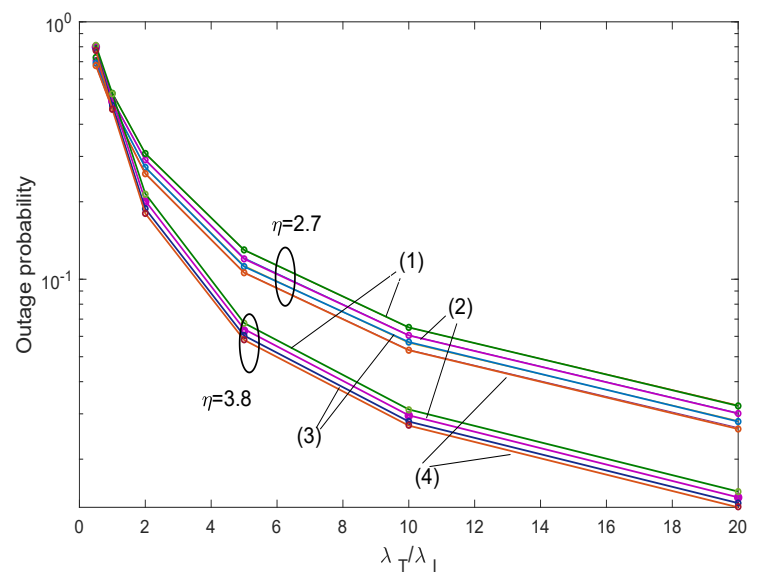

Fig. 2: Outage probability (11) for different generalized gamma fading distributions of useful and interfering links. $z=10 \mathrm{~dB}$, $P_{\mathrm{T}} / P_{\mathrm{I}}=10$. (1) - Nakagami- $m / \mathrm{GG}$ scenario; $m=1.7 ; \nu_{\mathrm{I}}=$ 6. (2) - Nakagami- $m /$ Nakagami- $m$ scenario; $m=1.7$. (3) GG/Nakagami- $m$ scenario; $m=1.7 ; \nu_{\mathrm{T}}=6$. (4) -Nakagami$m /$ Nakagami- $m$ scenario with $m_{\mathrm{T}}=3.5$ and $m_{\mathrm{I}}=0.7$.

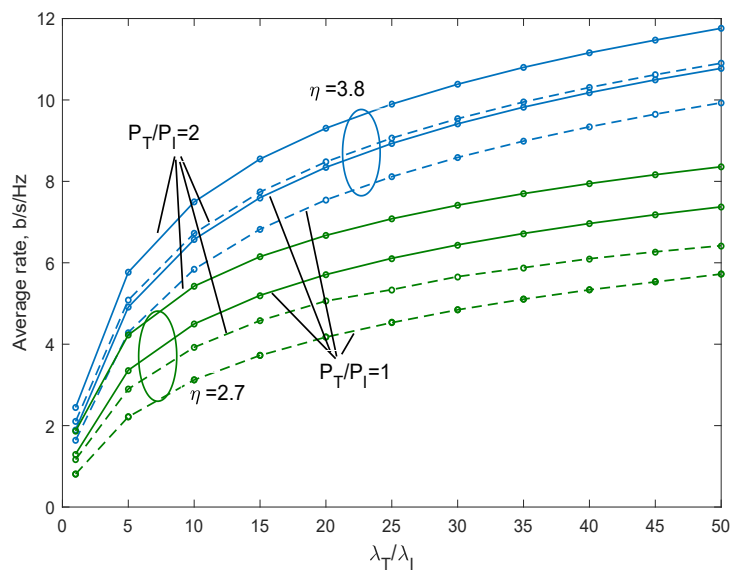

Fig. 3: Average rates for interference-limited scenarios. Solid lines represent estimates (13), and dotted lines represent estimates (12).

$B_{j}$, see (1), the Fox $H$-function reduces to the Meijer $G$ function, which is implemented in many modern software packages. This fact makes the results of this work quite general and convenient for practical purposes. The method of the $H$-function implementation via the Meijer $G$-function was applied in this work, and numerical evaluations were fast and efficient. The results of this work are general and applicable to arbitrary fading conditions.

\section{APPENDIX A \\ PROOF OF PROPOSITION 5}

We apply a capacity formula [20, eq. (2)]. The required MGFs of the useful signal and interference can be obtained from (3) with the respective parameters $K=\tilde{K}_{\text {us }}$ and $K=$ $\tilde{K}_{\text {int }}$. To derive an approximation, we use a change of va byriables $t=s^{\frac{2}{\eta}}$, and apply an identity formula $\frac{\left[1-\exp \left(-\tilde{K}_{\mathrm{us}} t\right)\right]}{t}=$ 


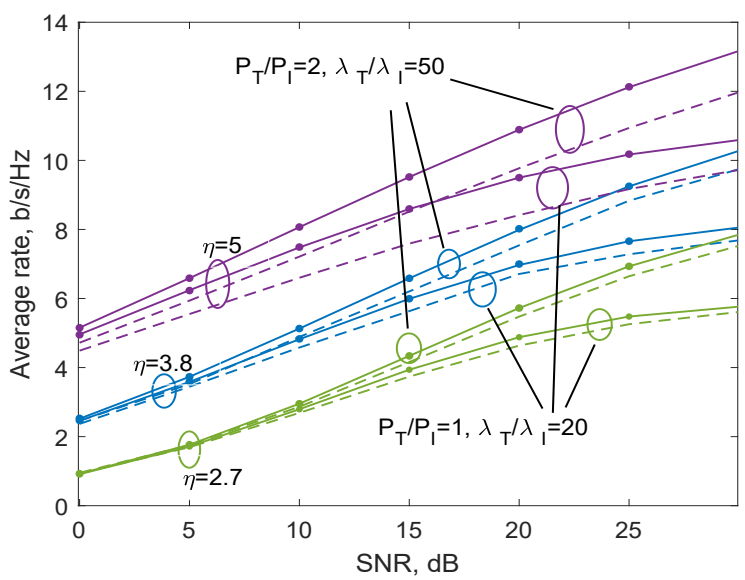

Fig. 4: Average rates (14). Useful and interfering links follow identical Nakagami- $m$ distributions with $m=2.7$. Solid and dashed lines represent exact and approximate estimates, respectively.

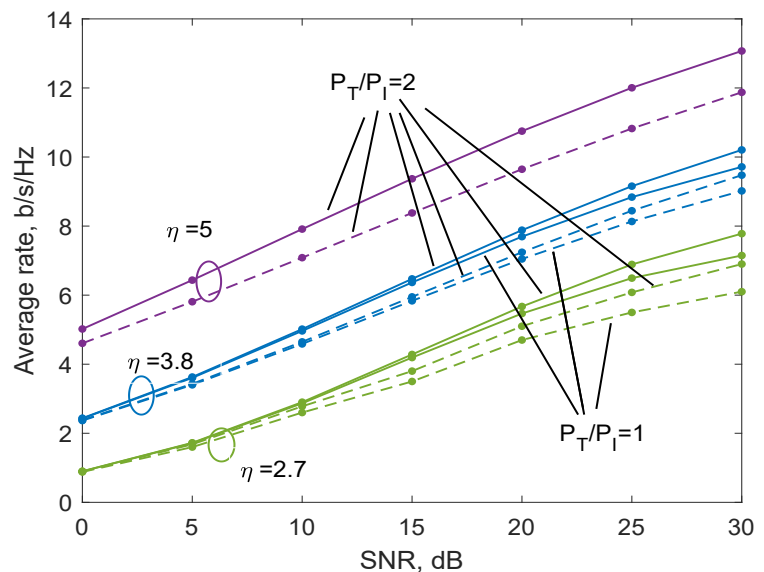

Fig. 5: Average rates for interference-plus-noise scenarios under identical Nakagami- $m$ fading for useful and interfering links; $m=1.3$. Solid lines represent estimates under non-orthogonal cooperative transmission (14), and dashed lines represent estimates under orthogonal cooperative transmission (15).

$-\tilde{K}_{\text {us }} \mathbb{E}_{1,2}\left(-\tilde{K}_{\text {us }} t\right)$ [18, eq. (ix)], a Laplace transform formula $\mathcal{L}\left\{E_{1,2}\left(-\tilde{K}_{\text {us }} t\right) ;\left\{t ; \tilde{K}_{\text {int }}\right\}\right\}=\frac{1}{\tilde{K}_{\text {int }}}{ }_{2} F_{1}\left(1,1,2,-\frac{\tilde{K}_{\text {us }}}{\tilde{K}_{\text {int }}}\right)$, [18, eq. (11.15)], [18, eq. (8.7)], as well as inequalities $\exp \left(-t^{\frac{\eta}{2}}\right) \geq \exp (-t)$ if $0<t \leq 1$ and $\exp \left(-t^{\frac{\eta}{2}}\right)<$ $\exp (-t)$ if $t>1$. Then one can obtain that (14) can be assessed as $\tilde{\mathcal{R}}_{\mathrm{n} / \mathrm{o}}=\frac{\eta \tilde{K}_{\mathrm{us}}}{2 \ln 2} \int_{0}^{\infty} \exp \left(-\tilde{K}_{\mathrm{int}} t\right) \mathbb{E}_{1,2}\left(-\tilde{K}_{\mathrm{us}} t\right)$ $\exp \left(-t^{\frac{\eta}{2}}\right) d t \approx \frac{\eta \tilde{K}_{\mathrm{us}}}{2 \ln 2\left(\tilde{K}_{\mathrm{int}}+1\right)}{ }_{2} F_{1}\left(1,1,2,-\frac{\tilde{K}_{\mathrm{us}}}{\tilde{K}_{\mathrm{int}}+1}\right) \stackrel{(a)}{=}$ $\frac{\eta}{2 \ln 2} \ln \left(1+\frac{\tilde{K}_{\mathrm{us}}}{\tilde{K}_{\mathrm{int}}+1}\right)$, where $(a)$ is due to [17, eq. (7.3.2.148)].

\section{APPENDIX B}

\section{ProOF OF PROPOSITION 5}

The MGF of interference can be defined via (3) with $K=$ $\tilde{K}_{\text {int }}$. In order to derive the MGF of the useful signal, we take into account that its PDF $f_{X_{\text {uso }}}(z)$ can be represented as $f_{X_{\mathrm{us}_{\mathrm{o}}}}(z) \stackrel{(a)}{=} \frac{2}{\eta} \Theta^{\frac{2}{\eta}} z^{-\frac{2}{\eta}-1} \exp \left[-\Theta^{\frac{2}{\eta}} z^{-\frac{2}{\eta}}\right] \stackrel{(b)}{=} \Theta^{-1}$ $\times H_{1,0}^{0,1}\left[\Theta^{-1} \not \mid\left(-\frac{\eta}{2}, \frac{\eta}{2}\right)\right]$, where (a) is due to [9], and (b) results from a representation of the exponential function via the Fox H-function [14, eq. (1.39)] and H-function properties [13, eqs. (2.3), (2.4)]. Then the MGF can be obtained via a Laplace transform formula [13, eq. (2.10)] as $\mathcal{M}_{X_{\mathrm{us}_{\mathrm{o}}}}(s)=$ $H_{0,2}^{2,0}\left[\Theta s \mid(0,1),\left(1, \frac{\eta}{2}\right)\right]$, and (15) follows from the capacity formula [20, eq. (2)].

\section{REFERENCES}

[1] T. Irnich, J. Kronander, Y. Selen, and G.Li, "Spectrum sharing scenarios and resulting technical requirements for 5G systems," in Proc. IEEE PIMRC, 2013, pp. 127-132.

[2] A. Gupta and R. K. Jha, "A survey of 5G network: Architecture and emerging technologies," IEEE Access, vol. 3, pp. 1206-1232, Jul. 2015.

[3] H. ElSawy, E. Hossain, and M. Haenggi, "Stochastic Geometry for Modeling, Analysis, and Design of Multi-Tier and Cognitive Cellular Wireless Networks: A Survey," IEEE Commun. Surv. and Tutor, vol. 15, pp. 996-1019, Third Quarter 2013.

[4] S. Toumpis and A. J. Goldsmith, "Capacity regions for wireless ad hoc networks," IEEE Trans. Inform. Theory, vol. 2, pp. 736-748, July 2003.

[5] P. Cardieri, "Modeling interference in wireless ad hoc networks," IEEE Commun. Surv. and Tutor., vol. 12, no. 4, pp. 551-572, 2010.

[6] J. Venkataraman, M. Haenggi, and O. Collins, "Shot noise models for outage and throughput analyses in wireless ad hoc networks," in Proc. IEEE MILCOM, pp. 1-7, 2006.

[7] P. C. Pinto and M. Z. Win, "Communication in a Poisson field of interferers - part I: Interference distribution and error probability," IEEE Trans. Wireless Commun., vol. 9, no. 7, pp. 2176-2186, July 2010.

[8] Y. M. Shobowale and K. A. Hamdi, "A unified model for interference analysis in unlicensed frequency bands," IEEE Trans. Wireless Commun., vol. 8, no. 8, pp. 4004-4013, Aug. 2009.

[9] N. Y. Ermolova and O. Tirkkonen, "Interference analysis in wireless networks operating over arbitrary fading channels with heterogeneous Poisson fields of transmitters and interferers," IEEE Signal Proces. Lett., vol. 24, pp. 1388-1392, Sept. 2017.

[10] M. K. Simon and M.-S. Alouini, Digital Communication over Fading Channels. Hoboken, NJ, USA: Wiley, 2005.

[11] M. D. Yacoub, "The $\kappa-\mu$ distribution and the $\eta-\mu$ distribution," IEEE Ant. and Propag. Mag., vol. 49, pp. 68-81, Feb. 2007.

[12] J. Hu, L.-L. Yang, and L. Hanzo, "Distributed cooperative social multicast aided content dissemination in random mobile networks," IEEE Trans. Veh. Techn., vol. 64, no. 7, pp. 3075-3089, July 2015.

[13] B. D. Carter and M. D. Springer, "The distribution of products, quotients and powers of independent H-function variates," SIAM J. Appl. Math. vol. 33, pp. 542-558, Dec. 1977.

[14] A. M. Mathai, R. K. Saxena, and H. J. Haubold. The H-Function, Theory and Applications. Springer, Amsterdam, 2006

[15] F. Yilmaz and M.-S. Alouini, "Product of the powers of generalized Nakagami-m variates and performance of cascaded fading channels," in Proc. IEEE GLOBECOM, pp. 1-8, 2009.

[16] K. Peppas, "A new formula for the average bit error probability of dualhop amplify-and-forward relaying systems over generalized shadowed fading channels," IEEE Wireless Commun. Lett., vol. 1, pp. 85-88, April 2012.

[17] A. P. Prudnikov, Y. A. Brychkov, and O. I. Marichev, Integrals and Series. New York, NY: Gordon and Breach, 1986

[18] H. J. Haubold, A. M. Mathai, and R.K. Saxena, "Mittag-Leffler functions and their applications, J. Appl. Math., 298628, 2011.

[19] M.-S. Alouini and A. Goldsmith, "Area spectral efficiency of cellular mobile radio systems," IEEE Trans. Veh. Techn., vol. 48, pp. 1047-1066, July 1999.

[20] K. A. Hamdi, "A useful lemma for capacity analysis of fading interference channels," IEEE Trans. Commun., vol. 58, pp. 411-416, Feb. 2010.

[21] T. S Rappaport, Wireless Communications: Principles and Practice, Upper Saddle River, NJ, USA: Prentice Hall, 2002, 2nd edition.

[22] V. A. Aalo, T. Piboongungon, and C.-D. Iskander, "Bit-error rate of binary digital modulation schemes in generalized gamma fading channels," IEEE Commun. Lett., vol. 9, pp. 139-141, Feb. 2005. 